\title{
DEGENERATION OF PSEUDO-LAPLACE OPERATORS FOR HYPERBOLIC RIEMANN SURFACES
}

\author{
LIZHEN JI
}

(Communicated by Dennis A. Hejhal)

\begin{abstract}
For finite volume, noncompact Riemann surfaces with their canonical hyperbolic metrics, there is a notion of pseudo-Laplace operators which include all embedded eigenvalues $\left(>\frac{1}{4}\right)$ of the Laplacian as a part of their eigenvalues. Similarly, we define pseudo-Laplace operators for compact hyperbolic Riemann surfaces with short geodesics. Then, for any degenerating family of hyperbolic Riemann surfaces $S_{l} \quad(l \geq 0)$, we show that normalized pseudoeigenfunctions and pseudoeigenvalues of $S_{l}$ converge to normalized pseudoeigenfunctions and pseudoeigenvalues of $S_{0}$ as $l \rightarrow 0$. In particular, normalized embedded eigenfunctions and their embedded eigenvalues of $S_{0}$ can be approximated by normalized pseudoeigenfunctions and pseudoeigenvalues of $S_{l}$ and $l \rightarrow 0$.
\end{abstract}

\section{INTRODUCTION}

Let $S$ be a noncompact, complete hyperbolic surface of finite area. Then the Laplacian of $S$ has continuous spectrum $\left[\frac{1}{4}+\infty\right)$ and discrete spectrum which may be embedded in the continuous part (see [12]). To study Weyl's asymptotic formula for the discrete spectrum, Lax and Phillips [9, Theorem 8.6] introduced an operator $\Delta_{a}$, which is called the pseudo-Laplacian by Colin de Verdière [4]. In [4] the pseudo-Laplacian was used to show, among other things, that, under generic compactly supported conformal perturbation of hyperbolic metrics, all the embedded eigenvalues $\left(\geq \frac{1}{4}\right)$ are destroyed. Pseudo-Laplacians are also useful in studying embedded eigenvalues for hyperbolic deformations. Using the pseudo-Laplacian, Deshoullers, Iwaniec, Phillips, and Sarnak [5, 11], showed that, by deforming certain arithmetic surfaces in a generic direction, infinitely many embedded eigenvalues are destroyed. Based on this, Phillips and Sarnak [10] conjectured that a generic hyperbolic surface has only finitely many embedded eigenvalues, contrary to a conjecture of Selberg [12].

Inspired by [4], we define the pseudo-Laplacian for hyperbolic surfaces with short geodesics. We believe that spectral degeneration of surfaces will help us understand spectra of noncompact surfaces, and pseudo-Laplacians are an important tool for studying degeneration. Actually, a preliminary version of this

Received by the editors June 30, 1992 and, in revised form, August 15, 1992.

1991 Mathematics Subject Classification. Primary 58G25, 58G18; Secondary 51M10.

Partially supported by NSF grant DMS 8907710. 
paper is used by Wolpert in [18], where he recently verified the above conjecture of Phillips and Sarnak for a special family of hyperbolic surfaces.

Now we give a detailed introduction to this paper. Let $S_{l}$ be a degenerating family of compact hyperbolic surfaces, i.e., there are several simple closed geodesics $\gamma_{1}(l), \ldots, \gamma_{m}(l)$ on $S_{l}$ whose lengths go to zero as $l \rightarrow 0$. Following [4], we define a pseudo-Laplacian $\Delta_{a}(l)$ for $S_{l}$. The operator $\Delta_{a}(l)$ acts on a subspace $\bar{H}\left(S_{l}\right) \subset L^{2}\left(S_{l}\right)$, which is defined by the vanishing of the zeroth Fourier coefficient in the pinching collars around the geodesics $\gamma_{1}(l), \ldots, \gamma_{m}(l)$ on $S_{l}$ (see $\S 2$ for details).

Definition 1.1. Eigenvalues and eigenfunctions of $\Delta_{a}(l)$ are called pseudoeigenvalues and pseudoeigenfunctions, respectively. Let $\left\{\lambda_{i}(l)\right\}_{i=1}^{\infty}$ denote the pseudoeigenvalues and $\left\{\psi_{i}(l)\right\}_{i=1}^{\infty}$ denote their corresponding orthonormal pseudoeigenfunctions.

For any $\lambda>0$, define a kernel function on $S_{l} \times S_{l}$,

$$
K_{\lambda}(z, w ; l)=\sum_{\lambda_{i}(l) \leq \lambda} \psi_{i}(l)(z) \psi_{i}(l)(w) .
$$

Further, to compare functions on $S_{0}$ and $S_{l}$, we would like to use the harmonic map of infinite energy from $S_{0}$ to $S_{l}$ constructed by Wolf [13]. In order to preserve the domains of the pseudo-Laplacians, i.e., to preserve the longitudes and meridians in the pinching collars and the cusps, we use the modified harmonic map by Wolpert [18, Chapter 2], which is denoted by $\pi_{l}: S_{0} \rightarrow S_{l}$. Intuitively speaking, the (modified) harmonic map opens up each node of $S_{0}$ into a simple closed geodesic of $S_{l}$ and is a homeomorphism from $S_{0}$ to $S_{l} \backslash\left\{\gamma_{1}(l), \ldots, \gamma_{m}(l)\right\}$ such that the pull-back of the $S_{l}$-hyperbolic metric converges in $C^{k}$-norm on compact subsets to the $S_{0}$-hyperbolic metric for any $k \in \mathbf{N}$. Then, we have the following

Theorem 1.2. (1) For any $i \geq 1, \lim _{l \rightarrow 0} \lambda_{i}(l)=\lambda_{i}(0)$.

(2) For any sequence $l_{j} \rightarrow 0$, let $\left\{\psi_{i}\left(l_{j}\right)\right\}_{i=1}^{\infty}$ be any choice of a complete system of orthonormal pseudoeigenfunctions on $S_{l_{j}}$. Then there is a subsequence $\left\{l_{j}^{\prime}\right\} \subset\left\{l_{j}\right\}$, such that $\lim _{j \rightarrow \infty} \pi_{l l_{j}}^{*} \psi_{i}\left(l_{j}^{\prime}\right)$ exists for all $i$. Let $\psi_{i}(0)=$ $\lim _{j \rightarrow \infty} \pi_{l_{j}}^{*} \psi_{i}\left(l_{j}^{\prime}\right)$. The limit functions $\left\{\psi_{i}(0)\right\}_{i=1}^{\infty}$ form a complete system of orthonormal pseudoeigenfunctions on $S_{0}$.

(3) For any $\lambda>0$ and $\lambda \notin \operatorname{Spec}\left(\Delta_{a}(0)\right), \lim _{l \rightarrow 0} K_{\lambda}\left(\pi_{l}(z), \pi_{l}(w) ; l\right)=$ $K_{\lambda}(z, w ; 0)$, where the convergence is uniform over compact subsets of $S_{0} \times S_{0}$.

Now for a noncompact surface $S_{0}$, embedded eigenvalues of the Laplacian are included among the pseudoeigenvalues (see Theorem 2.1). Then immediately, we have

Corollary 1.3. Let $\lambda(0) \geq \frac{1}{4}$ be an eigenvalue of $S_{0}$ with multiplicity $n$. Then, for any sequence $l_{j} \rightarrow 0$, there is a subsequence $\left\{l_{j}^{\prime}\right\} \subset\left\{l_{j}\right\}$ and normalized pseudoeigenfunctions $\psi_{i_{1}}\left(l_{j}^{\prime}\right), \ldots, \psi_{i_{n}}\left(l_{j}^{\prime}\right)$ on $S_{l_{j}^{\prime}}$, with pseudoeigenvalues $\lambda_{i_{1}}\left(l_{j}^{\prime}\right), \ldots, \lambda_{i_{n}}\left(l_{j}^{\prime}\right)$ such that, for $1 \leq k \leq n, \lim _{j \rightarrow \infty} \lambda_{i_{k}}\left(l_{j}^{\prime}\right)=\lambda(0)$ and $\lim _{j \rightarrow \infty} \pi_{l_{j}}^{*}\left(\psi_{i_{k}}\left(l_{j}^{\prime}\right)\right)$ exists. Further, the limit functions form an orthonormal basis of the $\lambda_{0}$ eigenspace of $S_{0}$.

In fact, we conjecture that this corollary holds for the eigenfunctions (of $\Delta(l)$ ) themselves. Pseudoeigenfunctions $\left\{\psi_{i}(l)\right\}$ of $S_{l}$ satisfy $\Delta(l) \psi_{i}(l)-\lambda_{i}(l) \psi_{i}(l)=$ 
0 , on $S_{l} \backslash \partial\left(\cup C_{l}(a)\right)$, where $\bigcup C_{l}(a)$ is the union of all pinching collars embedded in $S_{l}$ and $\Delta(l)$ is the Beltrami-Laplace operator (see $\S 2$ for details). For those pseudoeigenfunctions converging to embedded eigenfunctions, their zeroth Fourier coefficients in the pinching collars converge to zero as $l \rightarrow 0$, so cutting off the zeroth Fourier coefficient inside the pinching collars should not have much influence on these functions. It is conceivable that we can perturbate these pseudoeigenfunctions and recover some information about eigenfunctions of $S_{l}$, which approximate the embedded eigenfunctions of $S_{0}$ as $l \rightarrow 0$.

There are other motivations for studying degeneration of the pseudoLaplacians. When we take the whole $\left(\Delta(l), L^{2}\left(S_{l}\right)\right)$ space into consideration, the eigenvalues of $S_{l}$ cluster at every point of $\left[\frac{1}{4}, \infty\right)$ as $l \rightarrow 0$. During the degeneration, the dominant role is played by the part converging to the continuous spectrum of $S_{0}$. But this phenomenon is independent of the global geometry of $S_{l}$ and, instead, only depends on the lengths of the pinching geodesics $\left\{\gamma_{1}(l), \ldots, \gamma_{m}(l)\right\}$ (see [14,7 and 8]). It is expected that the pseudo-Laplacian $\Delta_{a}(l)$ can carry global information about $S_{l}$ during degeneration. Furthermore, from [8] and [16], we can distinguish between eigenfunctions which converge to generalized eigenfunctions of $S_{0}$ (they are given by Eisenstein series) and other eigenfunctions which converge to embedded eigenfunctions of $S_{0}$. An open problem is to characterize the subspace of $L^{2}\left(S_{l}\right)$ which converges to the subspace of $L^{2}\left(S_{0}\right)$ spanned by all the embedded eigenfunctions of $S_{0}$ as $l \rightarrow 0$.

The organization of the rest of this paper is as follows. In $\S 2$ we define pseudoLaplacians on $S_{l}$ and characterize them in terms of Fourier expansions. In $\S 3$ we prove Theorem 1.2. The key is to show that the limit pseudoeigenfunctions are linearly independent. Unlike the case for eigenfunctions of eigenvalues $<\frac{1}{4}$, there is no easy way to prove the linear independence. Instead we prove a stronger statement that they are orthonormal. In order to do this, we need to show that no mass of pseudoeigenfunctions of $S_{l_{j}}$ is lost deep inside the pinching collars during degeneration. The family $\left(\Delta_{a}(l), \overline{H_{a}}\left(S_{l}\right)\right) \quad(l \geq 0)$ is like a regular one, since we have removed the part of $L^{2}\left(S_{l}\right)$ which causes the clustering of eigenvalues of $S_{l}$ as $l \rightarrow 0$ (in [18], Wolpert shows that Kato's asymptotic perturbation theory applies to this family). To make this intuitive argument rigorous, we need the detailed study of the spectral degeneration of the collars around the pinching geodesics as we have worked out in $[8$, Theorem $1.5]$.

\section{Definitions OF PSEUdo-LAPLACIANS}

In this section, we will define pseudo-Laplacians and recall their basic properties. We start with the subspace of $L^{2}\left(S_{0}\right)$ where the pseudo-Laplacian acts. For $S_{0}$ a noncompact hyperbolic surface of finite area and $a \geq 0$, let $C_{0}(a)$ be a horocyclic neighbourhood of a puncture of $S_{0}$ with boundary length $e^{-a}$ and $U C_{0}(a)$ be the union of all such neighbourhoods in $S_{0}$. For any function $f$ on $S_{0}$, let $f_{0}(r, \theta)=f_{0}(r)$ be the zeroth Fourier coefficient of $f$ restricted to a horocyclic neighbourhood $C_{0}(a)$, where $(r, \theta)$ are the Fermi coordinates on $C_{0}(a)$. For $a>1$, define a subspace of $L^{2}\left(S_{0}\right)$,

$$
H_{a}(0)=\left\{f \in W^{2,1}\left(S_{0}\right) \mid f_{0}(r, \theta)=0 \text { for each }(r, \theta) \in \bigcup C_{0}(a)\right\},
$$


where $W^{2,1}\left(S_{0}\right)$ is the Sobolev space of $S_{0}$,

$$
W^{2,1}\left(S_{0}\right)=\left\{\left.f \in L^{2}\left(S_{0}\right)\left|\int\right| \nabla f\right|^{2}+f^{2}<\infty\right\} .
$$

Similarly, for $S_{l}$ with $l>0$, let $\cup C_{l}(a)$ be the union of all the pinching collars on $S_{l}$ with boundary length $e^{-a}$. The zeroth Fourier coefficient of any function $f$ on $\cup C_{l}(a)$ is also denoted by $f_{0}(r, \theta)$, where $(r, \theta)$ are the Fermi coordinates on the pinching collars. Then, for $a>1$, define a subspace of $L^{2}\left(S_{l}\right)$,

$$
H_{a}(l)=\left\{f \in W^{2,1}\left(S_{l}\right) \mid f_{0}(r, \theta)=0 \text { for }(r, \theta) \in \bigcup C_{l}(a)\right\},
$$

where $W^{2,1}\left(S_{l}\right)$ is the Sobolev space associated to $S_{l}$ as above.

Now we define the pseudo-Laplacians. The space $H_{a}(0)$ is a closed subspace of $W^{2,1}\left(S_{0}\right)$. The symmetric form $D_{0}[f, g]=\int_{S_{0}} \nabla_{0} f \nabla_{0} g$ restricts to $H_{a}(0)$ and induces by Friedrich's procedure a selfadjoint operator $\Delta_{a}(0)$ on $\overline{H_{a}(0)} \subset$ $L^{2}\left(S_{0}\right)$, which is called the pseudo-Laplacian of $S_{0}$. (See [4, Theorems 1,2 , and $5 ; 9$, pp. 206-208; 15].)

Theorem 2.1 (Colin de Verdière et al. [4, Theorems 1, 2, and 5]). (1) The domain of $\Delta_{a}(0)$ consists of those $f \in H_{a}(0)$ such that $\Delta(0) f-\alpha \delta_{a}(r) \in L^{2}\left(S_{0}\right)$ for some $\alpha \in \mathbf{C}$, and then $\Delta_{a}(0) f$ is defined by $\Delta_{a}(0) f=\Delta(0) f-\alpha \delta_{a}(r)$, where $\delta_{a}(r)$ is the Dirac measure at $a, \Delta(0) f$ is in the sense of distribution, and $\Delta(0)$ is the Laplacian of $S_{0}$.

(2) The operator $\Delta_{a}(0)$ is selfadjoint with compact resolvents.

(3) The spectrum of $\Delta_{a}(0)$ is discrete and is the union with appropriate multiplicity of the following two sequences:

(a) $\left\{\lambda_{i}\right\}$, where $\lambda_{i}$ is an eigenvalue of $\Delta(0)$ on $S_{0}$ with an associated cuspidal eigenfunction $\varphi_{i}$ and the associated pseudoeigenfunction of $\Delta_{a}(0)$ is also $\varphi_{i}$;

(b) $\left\{\mu_{j}(a)\right\}$, coming from Eisenstein series, roughly a discrete approximation to the continuous spectrum $\left[\frac{1}{4}, \infty\right)$ of $S_{0}$.

By the same procedure, we get a selfadjoint operator $\Delta_{a}(l)$ on $\overline{H_{a}(l)} \in L^{2}\left(S_{l}\right)$ for $S_{l}$ with $l>0$, and call it the pseudo-Laplacian of $S_{l}$. The operator has the following properties (see [9, $\S 8$ pp. 206-208] for proofs).

Proposition 2.2. (1) The domain of $\Delta_{a}(l)$ and its action are analogs of $\Delta_{a}(0)$ in Theorem 2.1.

(2) The operator $\Delta_{a}(l)$ is selfadjoint with compact resolvents.

Proposition 2.3. For $l \geq 0$, let $\psi(l)$ be any eigenfunction of $\Delta_{a}(l)$ with eigenvalue $\lambda(l)$. Then it can be characterized as follows:

(1) For $z \in S_{1} \backslash \partial\left(\bigcup C_{l}(a)\right),\left(\Delta_{a}(l)-\lambda(l)\right) \psi(l)(z)=0$.

(2) Let $\psi_{0}(l)$ be the zeroth Fourier coefficient of $\psi(l)$ in the collars. Then $\psi_{0}(l)(r)=0$ for $(r, \theta) \in \bigcup C_{l}(a)$, i.e., inside the collars, but $\psi_{0}(l)$ is continuous and possibly not differentiable across the boundary $\partial\left(\bigcup C_{l}(a)\right) \subset S_{l}$.

(3) The function $\psi(l)-\psi_{0}(l)$ on $\bigcup C_{l}(0)$ is smooth across $\partial\left(\bigcup C_{l}(a)\right) \subset S_{l}$. 


\section{Proof of Theorem 1.2}

Before proving Theorem 1.2, we establish the following lemma.

Lemma 3.1. For any $i \geq 1$, any sequence $l_{j} \rightarrow 0$, and any normalized pseudoeigenfunction $\psi_{i}\left(l_{j}\right)$ of $\Delta_{a}\left(l_{j}\right)$ with pseudoeigenvalue $\lambda_{i}\left(l_{j}\right)$, assume that $\overline{\lim }_{j \rightarrow 0} \lambda_{i}\left(l_{j}\right)<+\infty$. Then there is a subsequence $\left\{l_{j}^{\prime}\right\} \subset\left\{l_{j}\right\}$ such that:

(1) The limit $\lim _{j \rightarrow \infty} \lambda_{i}\left(l_{j}^{\prime}\right)$ exists, and the limit is denoted by $\lambda_{i}^{\wedge}(0)$.

(2) The functions $\pi_{l_{j}^{\prime}}^{*}\left(\psi_{i}\left(l_{j}^{\prime}\right)\right)$ converge uniformly over compact subsets of $S_{0}$ to some function $\psi_{i}^{\wedge}(0)$ on $S_{0}$, which satisfies $\left(\Delta(0)-\lambda_{i}^{\wedge}(0)\right) \psi_{i}^{\wedge}(0)(z)=0$ for $z \in S_{0} \backslash \partial\left(\bigcup C_{0}(a)\right)$. The function $\psi_{i}^{\wedge}(0)$ is an eigenfunction of $\Delta_{a}(0)$ with eigenvalue $\lambda_{i}^{\wedge}(0)$.

Remark. Actually, $\psi_{i}^{\wedge}(0)$ has $L^{2}$-norm 1 (see Step 2 in the proof of Theorem 1.2).

Proof. To begin with, we have $\Delta\left(l_{j}\right) \psi_{i}\left(l_{j}\right)-\lambda_{i}\left(l_{j}\right) \psi_{i}\left(l_{j}\right)=0$ on $S_{l_{j}} \backslash \partial\left(\bigcup C_{l_{j}}(a)\right)$ and $\left\|\psi_{i}\left(l_{j}\right)\right\|_{L^{2}\left(S_{l_{j}}\right)}=1$. Since the zeroth Fourier term of $\psi_{i}\left(l_{j}\right)$ vanishes along $\partial\left(C_{l}(a)\right)$, using integration by parts, we get

$$
\int_{S_{l_{j}}}\left|\nabla \psi_{i}\left(l_{j}\right)\right|^{2}=\lambda_{i}\left(l_{j}\right) \int_{S_{l_{j}}}\left|\psi_{i}\left(l_{j}\right)\right|^{2}=\lambda_{i}\left(l_{j}\right) .
$$

Then, by regularity theory [6, Theorems 8.8 and 8.9] and the convergence of the modified harmonic maps, for any compact subset $K \subset S_{0} \backslash \partial\left(\cup C_{0}(a)\right)$ and $k \in \mathbf{N}$, there exists a constant $C=C(K, k)$ such that

$$
\left\|\pi_{l_{j}}^{*} \psi_{i}\left(l_{j}\right)\right\|_{W^{k, 2}(K)} \leq C .
$$

Take an exhaustion of $S_{0} \backslash \partial\left(\bigcup C_{0}(a)\right)$ by compact subsets. Then, by Sobolev's embedding theorem [1, Theorem 5.4] and a diagonal argument, there is a subsequence $\left\{l_{j}^{\prime}\right\} \subset\left\{l_{j}\right\}$ such that:

(1) The limit $\lim _{j \rightarrow \infty} \lambda_{i}\left(l_{j}^{\prime}\right)$ exists, and the limit is denoted by $\lambda_{i}^{\wedge}(0)$.

(2) The function $\pi_{l_{j}^{\prime}}^{*}\left(\psi_{i}\left(l_{j}^{\prime}\right)\right)$ converges uniformly over compact subsets of $S_{0} \backslash \partial\left(\bigcup C_{0}(a)\right)$ to some function $\psi_{i}^{\wedge}(0)$.

We have to prove the uniform convergence across the boundary $\partial\left(\cup C_{0}(a)\right)$. In each pinching collar $C_{l}(0)$ of $S_{l}$, for any function $f$ on $C_{l}(0)$, let

$$
f(r, \theta)=\alpha_{0}(r)+\sum_{n \neq 0} \alpha_{n}(r) e^{2 \pi n i \theta}
$$

be the Fourier expansion of $f$ with respect to $\theta$. Then $f_{0}(r, \theta)=\alpha_{0}(r)$ is the zeroth Fourier term of $f$. Define $f_{1}(r, \theta)=\sum_{n \neq 0} \alpha_{n}(r) e^{2 \pi n i \theta}$ to be the remaining summation. Note that we only decompose $f=f_{0}+f_{1}$ on the collar $C_{l}(0)$. We now study the convergence of $\psi_{i, 1}\left(l_{j}\right)$ (the nonzero Fourier term of $\psi_{i}\left(l_{j}\right)$ ) and $\psi_{i, 0}\left(l_{j}\right)$ (the zero Fourier term) inside the collar $C_{l}(0)$.

I. The nonzero Fourier terms. Note that $\left(\Delta\left(l_{j}\right)-\lambda_{i}\left(l_{j}\right)\right) \psi_{i, 1}\left(l_{j}\right)=0$ on $C_{l}(0)$, $\int_{C_{l}(0)}\left|\psi_{i, 1}\left(l_{j}\right)\right|^{2} \leq 1$, and

$$
\int_{C_{l}(0)}\left|\nabla \psi_{i, 1}\left(l_{j}\right)\right|^{2} \leq \int_{S_{l}}\left|\nabla \psi_{i}\left(l_{j}\right)\right|^{2}=\lambda_{i}(l) .
$$


Then by the same argument as above, there is a subsequence of $\left\{l_{j}^{\prime}\right\}$, still denoted by $\left\{l_{j}^{\prime}\right\}$, such that $\pi_{l_{j}^{\prime}}^{*}\left(\psi_{i, 1}\left(l_{j}^{\prime}\right)\right)$ converges uniformly over compact subsets of $\cup C_{0}(0)$ to $\psi_{i, 1}^{\wedge}(0)$. (The limit function agrees with the nonzero Fourier terms of the limit obtained above!) In particular, the convergence of $\pi_{l_{j}^{\prime}}^{*}\left(\psi_{i, 1}\left(l_{j}^{\prime}\right)\right)$ to $\psi_{i, 1}^{\wedge}(0)$ is uniform across the boundary $\partial\left(\bigcup C_{0}(a)\right)$.

II. The zero Fourier term. Since $\lim _{j \rightarrow \infty} \pi_{l_{j}^{\prime}}^{*}\left(\psi_{i}\left(l_{j}^{\prime}\right)\right)=\psi_{i}^{\wedge}(0)$ uniformly over $\bigcup C_{0}\left(\frac{1}{2} a\right) \backslash C_{0}\left(\frac{1}{4} a\right)$ and $\lim _{j \rightarrow \infty} \pi_{l_{j}^{\prime}}^{*}\left(\psi_{i, 1}\left(l_{j}^{\prime}\right)\right)=\psi_{i, 1}^{\wedge}(0)$ uniformly over $\bigcup C_{0}\left(\frac{1}{2} a\right) \backslash C_{0}\left(\frac{1}{4} a\right)$, it follows that $\lim _{j \rightarrow \infty} \pi_{l_{j}^{\prime}}^{*}\left(\psi_{i, 0}\left(l_{j}^{\prime}\right)\right)=\psi_{i, 0}^{\wedge}(0)$ uniformly over $\bigcup C_{0}\left(\frac{1}{2} a\right) \backslash C_{0}\left(\frac{1}{4} a\right)$. Notice that $\psi_{i, 0}(l)$ satisfies the ordinary differential equation, $\left(\Delta(l ; 0)-\lambda_{i}(l)\right) \psi_{i, 0}(l)=0$, where $\Delta(l ; 0)$ is the restriction of $\Delta_{l}$ to the subspace of rotationally invariant functions on $C_{l}(0) \subset S_{l}$, and thus it is a second-order ordinary differential operator in $r$. Since $\Delta(l ; 0)$ approaches $\Delta(0 ; 0)$ as ordinary differential operators, it follows from the stability of the initial value problem for ordinary differential equations that $\lim _{j \rightarrow \infty} \pi_{l_{j}^{\prime}}^{*}\left(\psi_{i, 0}\left(l_{j}^{\prime}\right)\right)$ $=\psi_{i, 0}^{\wedge}(0)$ uniformly over $\bigcup C_{0}(0) \backslash C_{0}(2 a)$, in particular, uniformly across the boundary $\partial\left(\cup C_{0}(a)\right)$.

By the above discussions, it is clear that $\lim _{j \rightarrow \infty} \pi_{l_{j}^{\prime}}^{*}\left(\psi_{i}\left(l_{j}^{\prime}\right)\right)=\psi_{i}^{\wedge}(0)$ uniformly over compact subsets of $S_{0}$. The limit function $\psi_{i}^{\wedge}(0)$ satisfies $\left(\Delta(0)-\lambda_{i}^{\wedge}(0)\right) \psi_{i}^{\wedge}(0)=0$ on $S_{0} \backslash \partial\left(\bigcup C_{0}(a)\right)$ and $\left\|\psi_{i}^{\wedge}(0)\right\|_{L^{2}\left(S_{0}\right)} \leq 1$.

Now we show that $\psi_{i}^{\wedge}(0)$ satisfies the vanishing condition in each cusp of $S_{0}$. Since $\lim _{j \rightarrow \infty} \pi_{l_{j}^{\prime}}^{*}\left(\psi_{i, 0}\left(l_{j}^{\prime}\right)=\psi_{i, 0}^{\wedge}(0)\right.$, then, for $(r, \theta) \in \bigcup C_{0}(a)$,

$$
\psi_{i, 0}^{\wedge}(r)=\lim _{j \rightarrow \infty} \pi_{l_{j}^{\prime}}^{*} \psi_{i, 0}\left(l_{j}^{\prime}\right)=\lim _{j \rightarrow \infty} 0=0
$$

Furthermore, since $\lim _{j \rightarrow \infty} \pi_{l_{j}^{\prime}}^{*} \psi_{i, 1}\left(l_{j}^{\prime}\right)=\psi_{i, 1}^{\wedge}(0)$ and $\psi_{i, 1}$ is smooth across $\partial\left(\bigcup C_{l}(a)\right) \subset S_{l}$, it is clear that $\psi_{i}^{\wedge}(0)-\psi_{i, 0}^{\wedge}(0)=\psi_{i, 1}^{\wedge}(0)$ is smooth across $\partial\left(\bigcup C_{0}(a)\right) \subset S_{0}$. Therefore, by Proposition 2.3 , the limit function $\psi_{i}^{\wedge}(0)$ is a pseudoeigenfunction of $\Delta_{a}(0)$ with pseudoeigenvalue $\lambda_{i}^{\wedge}(0)$.

Proof of Theorem 1.2. The proof is divided into three steps:

1. Show that for $i \geq 1, \varlimsup_{\lim _{l \rightarrow 0}} \lambda_{i}(l) \leq \lambda_{i}(0)$.

2. Show that for $i \geq 1, \varliminf_{l \rightarrow 0} \lambda_{i}(l) \geq \lambda_{i}(0)$ and, for $1 \leq k \leq i,\left\langle\psi_{i}^{\wedge}(0)\right.$, $\left.\psi_{k}^{\wedge}(0)\right\rangle=\delta_{i k}$.

3. Show that for $\lambda>0$ and $\lambda \notin \operatorname{Spec}\left(\Delta_{a}(0)\right), \lim _{l \rightarrow 0} K_{\lambda}\left(\pi_{l}(z), \pi_{l}(w) ; l\right)=$ $K_{\lambda}(z, w ; 0)$.

Step 1. For any $i \geq 1$ and $0<\delta<a$, let $f_{1}(0), \ldots, f_{i}(0)$ be the first $i$ orthonormal pseudoeigenfunctions of $\Delta_{a-\delta}(0)$ with pseudoeigenvalues $\lambda_{1}(0, \delta), \ldots, \lambda_{i}(0, \delta)$ on $S_{0}$. For any $\rho>a$, let $\eta_{\rho}$ be a cut-off function on $S_{0}, \eta_{\rho} \equiv 1$ on $S_{0} \backslash \cup C_{0}(\rho), \eta_{\rho} \equiv 0$ on $\bigcup C_{0}(\rho+1)$, and $\left|\nabla_{0} \eta\right| \leq 2$. Note that $f_{1}(0), \ldots, f_{i}(0)$ have exponential decays along the cusps. We can assume $\eta_{\rho} f_{1}(0), \ldots, \eta_{\rho} f_{i}(0)$ are linearly independent for $\rho \gg 1$. Define for $1 \leq k \leq i, f_{k}(l)=\left(\pi^{-1}\right)_{l}^{*}\left(\eta_{\rho} f_{k}(0)\right)$. Then $f_{1}(l), \ldots, f_{k}(l)$ are linearly inde- 
pendent and lie in the domain of $\Delta_{a}(l)$ when $l$ is small enough. Let

$$
\varepsilon(\rho)=\int_{\bigcup C_{0}(\rho) \backslash C_{0}(\rho+1)} \sum_{1}^{i}\left|\nabla f_{k}(l)\right|^{2}+\left|f_{k}(l)\right|^{2} .
$$

Then $\lim _{\rho \rightarrow \infty} \varepsilon(\rho)=0$. For any constants $a_{k}$ with $\sum_{1}^{i} a_{k}^{2}=1$, we have

$$
\begin{gathered}
\int_{S_{l}}\left|\nabla \sum_{1}^{i} a_{k} f_{k}(l)\right|^{2} \leq(1+\delta(l))\left(\lambda_{i}(0, \delta)+\varepsilon(\rho)\right), \\
\int_{S_{l}}\left|\sum_{1}^{i} a_{k} f_{k}(l)\right|^{2}=\left(1+\delta^{\prime}(l)\right)\left(1-\varepsilon^{\prime}(\rho)\right),
\end{gathered}
$$

where $\varepsilon(\rho), \varepsilon^{\prime}(\rho) \rightarrow 0$ as $\rho \rightarrow \infty$, and $\delta(l) \quad \delta^{\prime}(l) \rightarrow 0$ when $l \rightarrow 0$, and $\rho$ is fixed. Then by the Mini-Max principle (see [2, Chapter 1]), we have that

$$
\varlimsup_{l \rightarrow 0} \lambda_{i}(l) \leq\left(\lambda_{i}(0, \delta)+\varepsilon(\rho)\right) \frac{1}{1-\varepsilon^{\prime}(\rho)} .
$$

Let $\rho \rightarrow \infty$. We get that, for $i \geq 1, \overline{\lim } l \rightarrow 0 \lambda_{i}(l) \leq \lambda_{i}(0, \delta)$. Since the pseudoeigenvalues of $\Delta_{a}(0)$ depend continuously on $a$, let $\delta \rightarrow 0$. We get that, for $i \geq 1, \varlimsup_{\lim _{l \rightarrow 0}} \lambda_{i}(l) \leq \lambda_{i}(0)$.

Step 2. We use induction on $i$ in this case. For $i=1$, choose a sequence $l_{j} \rightarrow 0$ such that $\lim _{j \rightarrow \infty} \lambda_{1}\left(l_{j}\right)=\varliminf_{l \rightarrow 0} \lambda_{1}(l)$. By Lemma 3.1, there is a subsequence $\left\{l_{j}^{\prime}\right\} \subset\left\{l_{j}\right\}$ such that

$$
\lim _{j \rightarrow \infty} \lambda_{1}\left(l_{j}^{\prime}\right)=\lambda_{1}^{\wedge}(0), \quad \lim _{j \rightarrow \infty} \pi_{l_{j}^{\prime}}^{*}\left(\psi_{1}\left(l_{j}^{\prime}\right)\right)=\psi_{1}^{\wedge}(0) .
$$

We want to show that

$$
\left\langle\psi_{1}^{\wedge}(0), \psi_{1}^{\wedge}(0)\right\rangle=1 .
$$

In particular, the function $\psi_{1}^{\wedge}(0) \neq 0$, and $\lambda_{1}^{\wedge}(0)$ is a pseudoeigenvalue of $\Delta_{a}(0)$; in which case, $\lambda_{1}^{\wedge}(0) \geq \lambda_{1}(0)$ and, by the arbitrary choice of $\left\{l_{j}\right\}$, $\varliminf_{l \rightarrow 0} \lambda_{1}(l) \geq \lambda_{1}(0)$. Then by Step 1 we get $\lim _{l \rightarrow 0} \lambda_{1}(l)=\lambda_{1}(0)$ and $\psi_{1}^{\wedge}(0)$ is a normalized pseudoeigenfunction of $\Delta_{a}(0)$ with pseudoeigenvalue $\lambda_{1}(0)$.

Now we prepare to show that $\left\langle\psi_{1}^{\wedge}(0), \psi_{1}^{\wedge}(0)\right\rangle=1$. First we recall spectral degeneration of the pinching collars. Let $\left\{g_{i}(l)\right\}_{i=1}^{\infty}$ be the complete system of orthonormal Dirichlet eigenfunctions with eigenvalues $\left\{\lambda_{i}^{*}(l)\right\}_{i=1}^{\infty}$ of $\bigcup C_{l}(a)$ except the zeroth mode $\left(\Delta_{l}(0), L_{0}^{2}\left(\bigcup C_{l}(a)\right)\right)$ (that is, except rotationally invariant eigenfunctions) (see [8]). Since each pinching collar is symmetric with respect to its core (pinching) geodesic, we can further assume that, for $l>0$, $\left|g_{i}(l)\right|(i \geq 1)$ are symmetric with respect to its core pinching geodesic in each collar. Then we have

Theorem 3.2 [8, Theorem 1.5]. (1) For all $i \geq 1, \lim _{l \rightarrow 0} \lambda_{2 i}^{*}(l)=\lim _{l \rightarrow 0} \lambda_{2 i-1}^{*}(l)$ $=\lambda_{i}^{*}(0)$; in particular, $\left\{\lambda_{i}^{*}(l)\right\}_{i=1}^{\infty}$ does not cluster at any finite point as $l \rightarrow 0$.

(2) For all $i \geq 1$,

$$
\lim _{l \rightarrow 0} \pi_{l}^{*}\left(g_{2 i-1}(l)\right)^{2}=\frac{1}{2} g_{i}(0)^{2}, \quad \lim _{l \rightarrow 0} \pi_{l}^{*}\left(g_{2 i}(l)\right)^{2}=\frac{1}{2} g_{i}(0)^{2}
$$

uniformly over compact subsets of $\cup C_{0}(a)$. 
Note that the geometric limit of $C_{l}(a)$ as $l \rightarrow 0$ is a pair of cusps $C_{0}(a) \cup$ $C_{0}(a)$. Since we normalize $g_{i}(0)$ to be of $L^{2}$-norm 1 on each cusp $C_{0}(a)$, there is a factor $\frac{1}{2}$ appearing in the right-hand side of the above equations. Theorem 3.2 means intuitively that none of the $g_{i}(l)$ lose mass inside the collars as $l \rightarrow 0$. More precisely,

$$
\lim _{\rho \rightarrow \infty} \varlimsup_{l \rightarrow 0} \int_{\bigcup C_{l}(\rho)}\left|g_{i}(l)\right|^{2}=0 .
$$

Let $\xi$ be a cut-off function on $S_{l}, \xi \equiv 1$ on $\bigcup C_{l}(a+1)$, and $\xi \equiv 0$ on $S_{l} \backslash \bigcup C_{l}(a),|\nabla \xi| \leq 2$. Consider the function $\xi \psi_{1}\left(l_{j}^{\prime}\right)$ on $S_{l_{j}^{\prime}}$. Let

$$
\xi \psi_{1}\left(l_{j}^{\prime}\right)=\sum_{n=1}^{\infty} a_{n}\left(l_{j}^{\prime}\right) g_{n}\left(l_{j}^{\prime}\right)
$$

be the Fourier expansion in terms of $\left\{g_{n}\left(l_{j}^{\prime}\right)\right\}$, where $a_{n}\left(l_{j}^{\prime}\right)=\left\langle\xi \psi_{1}\left(l_{j}\right), g_{n}\left(l_{j}^{\prime}\right)\right\rangle$. Then

$$
\sum_{n=1}^{\infty} a_{n}^{2}\left(l_{j}^{\prime}\right)=\left\langle\xi \psi_{1}\left(l_{j}^{\prime}\right), \xi \psi_{1}\left(l_{j}^{\prime}\right)\right\rangle
$$

For $N>1$, define

$$
\delta(N)=\varlimsup_{l_{j}^{\prime} \rightarrow 0} \sum_{n \geq N} a_{n}^{2}\left(l_{j}^{\prime}\right) .
$$

Then we have the following

Claim. With the above notation, $\lim _{N \rightarrow \infty} \delta(N)=0$.

Proof. Suppose instead that $\delta(N) \geq c_{0}>0$ for $N \gg 1$. By Lemma 3.2, $\left\{\lambda_{n}^{*}(l)\right\}$ does not accumulate at any finite point; therefore,

$$
\lim _{N \rightarrow \infty} \lambda_{N}^{*}(l)=\infty
$$

uniformly for $0 \leq l \leq \alpha$, where $\alpha$ is a small fixed positive constant. Now we compute $\left\langle\Delta\left(l_{j}^{\prime}\right)\left(\xi \psi_{1}\left(l_{j}^{\prime}\right)\right), \xi \psi_{1}\left(l_{j}^{\prime}\right)\right\rangle$. For any $N>0$, by the Fourier expansion (equation (2)), we get

$$
\begin{gathered}
\left\langle\Delta\left(l_{j}^{\prime}\right)\left(\xi \psi_{1}\left(l_{j}^{\prime}\right)\right), \xi \psi_{1}\left(l_{j}^{\prime}\right)\right\rangle \geq \sum_{n \geq N} \lambda_{n}^{*}\left(l_{j}^{\prime}\right) a_{n}^{2}\left(l_{j}^{\prime}\right), \\
\varlimsup_{l_{j}^{\prime} \rightarrow 0}\left\langle\Delta\left(l_{j}^{\prime}\right)\left(\xi \psi_{1}\left(l_{j}^{\prime}\right)\right), \xi \psi_{1}\left(l_{j}^{\prime}\right)\right\rangle \geq \varliminf_{l_{j}^{\prime} \rightarrow 0} \lambda_{N}^{*}\left(l_{j}^{\prime}\right) \varlimsup_{l_{j}^{\prime} \rightarrow 0} \sum_{n \geq N} \alpha_{n}^{2}\left(l_{j}^{\prime}\right) \geq \varliminf_{l_{j}^{\prime} \rightarrow 0} \lambda_{N}^{*}\left(l_{j}^{\prime}\right) c_{0} .
\end{gathered}
$$

Let $N \rightarrow \infty$. From equation (3), we get

$$
\varliminf_{l_{j}^{\prime} \rightarrow 0}\left\langle\Delta\left(l_{j}^{\prime}\right)\left(\xi \psi_{1}\left(l_{j}^{\prime}\right)\right), \xi \psi_{1}\left(l_{j}^{\prime}\right)\right\rangle=+\infty .
$$

On the other hand, recall that

$$
\Delta\left(l_{j}^{\prime}\right) \psi_{1}\left(l_{j}^{\prime}\right)+\lambda_{1}\left(l_{j}^{\prime}\right) \psi_{1}\left(l_{j}^{\prime}\right)=0, \quad\left\|\psi_{1}\left(l_{j}^{\prime}\right)\right\|_{L^{2}\left(S_{l_{j}^{\prime}}\right)}=1
$$


and $\pi_{l_{j}^{\prime}}^{*}\left(\psi_{1}\left(l_{j}^{\prime}\right)\right)$ converges smoothly over compact subsets of $S_{0}$ to $\psi_{i}^{*}(0)$. Then we get immediately that

$$
\varlimsup_{l_{j}^{\prime} \rightarrow 0}\left|\left\langle\Delta\left(l_{j}^{\prime}\right) \xi \psi_{1}\left(l_{j}^{\prime}\right), \xi \psi_{1}\left(l_{j}^{\prime}\right)\right\rangle\right|<+\infty,
$$

which contradicts equation (4)! Thus we have proved the claim.

Now we are ready to prove $\left\langle\psi_{1}^{\wedge}(0), \psi_{1}^{\wedge}(0)\right\rangle=1$. By the claim above, for any $\delta>0$, there exists $N_{0}>0$ such that

$$
\int_{S_{l_{j}^{\prime}}}\left|\xi \psi_{1}\left(l_{j}^{\prime}\right)-\sum_{k=1}^{N_{0}} a_{n}\left(l_{j}^{\prime}\right) g_{n}\left(l_{j}^{\prime}\right)\right|^{2}<\delta .
$$

Using the above estimates and equation (2), we get

$$
\lim _{\rho \rightarrow \infty} \varlimsup_{j \rightarrow \infty} \int_{\bigcup C_{l_{j}^{\prime}}(\rho)}\left|\psi_{1}\left(l_{j}^{\prime}\right)\right|^{2} \leq \delta .
$$

By the arbitrary choice of $\delta>0$, it follows that

$$
\lim _{\rho \rightarrow \infty} \varlimsup_{j \rightarrow \infty} \int_{\bigcup C_{l_{j}^{\prime}}(\rho)}\left|\psi_{1}\left(l_{j}^{\prime}\right)\right|^{2}=0 .
$$

Note that $\left\langle\psi_{1}\left(l_{j}^{\prime}\right), \psi_{1}\left(l_{j}^{\prime}\right)\right\rangle=1$. Then it is clear that $\left\langle\psi_{1}^{\wedge}(0), \psi_{1}^{\wedge}(0)\right\rangle=1$. We denote the limit function $\psi_{1}^{\wedge}(0)$ by $\psi_{1}(0)$, which is a normalized pseudoeigenfunction of $L^{2}$-norm 1 .

Now we do the induction step for Step 2 of Theorem 1.2; that is, assume that, for $1 \leq k \leq i-1$,

$$
\lim _{l \rightarrow 0} \lambda_{k}(l)=\lambda_{k}(0), \quad \lim _{l_{j}^{\prime} \rightarrow 0} \pi_{l_{j}^{\prime}}^{*}\left(\psi_{k}\left(l_{j}^{\prime}\right)\right)=\psi_{k}(0)
$$

uniformly over compact subsets of $S_{0}$ and $\left\{\psi_{k}(0)\right\}_{1}^{i-1}$ are orthonormal pseudoeigenfunctions of $\Delta_{a}(0)$ with pseudoeigenvalues $\left\{\lambda_{k}(0)\right\}_{1}^{i-1}$. For fixed $i>1$, by Lemma 3.1 , there is a subsequence of $\left\{l_{j}^{\prime}\right\}$, still denoted by $\left\{l_{j}^{\prime}\right\}$, such that

$$
\lim _{l \rightarrow 0} \lambda_{i}(l)=\lambda_{i}^{\wedge}(0), \quad \lim _{l_{j}^{\prime} \rightarrow 0} \pi_{l_{j}^{\prime}}^{*}\left(\psi_{i}\left(l_{j}^{\prime}\right)\right)=\psi_{i}^{\wedge}(0)
$$

where the convergence is uniform over compact subsets of $S_{0}$ and $\psi_{i}^{\wedge}(0)$ is a pseudoeigenfunction of $\Delta_{a}(0)$ with pseudoeigenvalue $\lambda_{i}^{\wedge}(0)$. Next we show that

$$
\left\langle\psi_{k}(0), \psi_{i}^{\wedge}(0)\right\rangle=0, \quad 1 \leq k \leq i-1, \quad\left\langle\psi_{i}^{\wedge}(0), \psi_{i}^{\wedge}(0)\right\rangle=1 .
$$

One immediate consequence of the above orthonormality is that $\psi_{1}(0), \ldots$, $\psi_{i-1}(0), \psi_{i}^{\wedge}(0)$ are linearly independent; thus $\lambda_{i}^{\wedge}(0) \geq \lambda_{i}(0)$. Then by Step 1 , we have $\lambda_{i}^{\wedge}(0)=\lambda_{i}(0)$, and therefore $\lim _{l \rightarrow 0} \lambda_{i}(l)=\lambda_{i}(0)$.

At first, we prove, for $1 \leq k \leq i-1,\left\langle\psi_{k}(0), \psi_{i}^{\wedge}(0)\right\rangle=0$. Since $\left\langle\psi_{k}(0)\right.$, $\left.\psi_{k}(0)\right\rangle=1$, it is clear that

$$
1-\lim _{l_{j}^{\prime} \rightarrow 0} \int_{S_{l_{j}^{\prime}} \backslash \bigcup C_{l_{j}^{\prime}}(\rho)}\left|\psi_{k}\left(l_{j}^{\prime}\right)\right|^{2}=1-\int_{S_{0} \backslash \bigcup C_{0}(\rho)}\left|\psi_{k}(0)\right|^{2} .
$$


This implies that

$$
\lim _{\rho \rightarrow \infty} \varlimsup_{l_{j}^{\prime} \rightarrow 0} \int_{\bigcup C_{l_{j}^{\prime}}(\rho)}\left|\psi_{k}\left(l_{j}^{\prime}\right)\right|^{2}=\lim _{\rho \rightarrow \infty} \int_{\bigcup C_{0}(\rho)}\left|\psi_{k}(0)\right|^{2}=0 .
$$

Since, for $1 \leq k \leq i-1,\left\langle\psi_{k}\left(l_{j}^{\prime}\right), \psi_{i}\left(l_{j}^{\prime}\right)\right\rangle=0$,

$$
\begin{aligned}
\left|\int_{S_{0}} \psi_{k}(0) \psi_{i}^{\wedge}(0)\right|= & \left|\int_{S_{0}} \psi_{k}(0) \psi_{i}^{\wedge}(0)-\int_{S_{l_{j}^{\prime}}} \psi_{k}\left(l_{j}^{\prime}\right) \psi_{i}\left(l_{j}^{\prime}\right)\right| \\
\leq & \int_{\bigcup C_{0}(\rho)}\left|\psi_{k}(0) \psi_{i}^{\wedge}(0)\right|+\int_{\pi_{l_{j}^{\prime}}^{-1}\left(\bigcup C_{0}(\rho)\right)}\left|\psi_{k}\left(l_{j}^{\prime}\right) \psi_{i}\left(l_{j}^{\prime}\right)\right| \\
& +\int_{S_{0} \backslash \bigcup C_{0}(\rho)}\left|\psi_{k}(0) \psi_{i}(0)-\pi_{l_{j}^{\prime}}^{*}\left(\psi_{k}\left(l_{j}^{\prime}\right) \psi_{i}\left(l_{j}^{\prime}\right)\right) \frac{d \mu_{l_{j}^{\prime}}}{d \mu_{0}}\right| \\
\leq & \left(\int_{\bigcup C_{0}(\rho)} \psi_{k}^{2}(0)\right)^{1 / 2}+\left(\int_{\pi_{l_{j}^{\prime}}^{-1}\left(\bigcup C_{0}(\rho)\right)} \psi_{k}^{2}\left(l_{j}^{\prime}\right)\right)^{1 / 2} \\
& +\int_{S_{0} \backslash \bigcup C_{0}(\rho)}\left|\psi_{k}(0) \psi_{i}(0)-\pi_{l_{j}^{\prime}}^{*}\left(\psi_{k}\left(l_{j}^{\prime}\right) \psi_{i}\left(l_{j}^{\prime}\right)\right) \frac{d \mu_{l_{j}^{\prime}}}{d \mu_{0}}\right|,
\end{aligned}
$$

where $d \mu_{l}$ is the volume form of $S_{l}$. For each fixed $\rho$, the third term goes to zero as $j \rightarrow+\infty$. Then from equation (5), it follows that

$$
\left|\int_{S_{0}} \psi_{k}(0) \psi_{i}^{\wedge}(0)\right|=0 \text {. }
$$

This proves the orthogonality.

The proof that $\left\langle\psi_{i}^{\wedge}(0), \psi_{i}^{\wedge}(0)\right\rangle=1$ is exactly the same as for the case $i=1$ above. Therefore, $\psi_{1}(0), \ldots, \psi_{i-1}, \psi_{i}^{\wedge}(0)$ are orthonormal pseudoeigenfunctions of $\Delta_{a}(0)$ with pseudoeigenvalues $\lambda_{1}(0), \ldots, \lambda_{i}(0)$. Thus we can denote the limit function $\psi_{i}^{\wedge}(0)$ by $\psi_{i}(0)$ and finish the induction step on $i$. Finally, by a diagonal argument, we can choose a subsequence which satisfies the conditions in Theorem 1.2(2).

Step 3. By Step 2 , for any sequence $l_{j} \rightarrow 0$, there exists a subsequence $\left\{l_{j}^{\prime}\right\} \subset$ $\left\{l_{j}\right\}$ such that, for all $k \geq 1$,

$$
\lim _{l_{j}^{\prime} \rightarrow 0} \pi_{l_{j}^{\prime}}^{*}\left(\psi_{k}\left(l_{j}^{\prime}\right)\right)=\psi_{k}(0), \quad \lim _{l_{j}^{\prime} \rightarrow 0} \lambda_{k}(l)=\lambda_{k}(0) .
$$

Since $\lambda \notin \operatorname{Spec}\left(\Delta_{a}(0)\right), \lambda_{k}(0) \leq \lambda$ if and only if $\lambda_{k}(l) \leq \lambda$ for all small enough $l$. Therefore,

$$
\lim _{l_{j}^{\prime} \rightarrow 0} K_{\lambda}\left(\pi_{l_{j}^{\prime}}(z), \pi_{l_{j}^{\prime}}(w) ; l\right)=K_{\lambda}(z, w ; 0),
$$

where the convergence is uniform over compact subsets of $S_{0} \times S_{0}$. By the arbitrary choice of $\left\{l_{j}\right\}$, we get

$$
\lim _{l \rightarrow 0} K_{\lambda}\left(\pi_{l}(z), \pi_{l}(w) ; l\right)=K_{\lambda}(z, w ; 0),
$$

where the convergence is uniform over compact subsets of $S_{0} \times S_{0}$. 


\section{ACKNOWLEDGMENTS}

The author thanks Professor S. T. Yau for his encouragement and his interest in this paper and Professor M. Goresky for listening to the author's explanation of the paper and for his advice. The author also thanks Professor R. Wentworth for helpful conversations and Professor S. Stafford for careful proofreading. Finally, the author thanks an anonymous referee for his careful reading and kind suggestions.

\section{REFERENCES}

1. R. A. Adams, Sobolev spaces, Academic Press, New York, 1975.

2. I. Chavel, Eigenvalues in Riemann geometry, Academic Press, New York, 1984.

3. Y. Colin de Verdière, Pseudo-Laplaciens. I, Ann. Inst. Fourier (Grenoble) 32 (1982), 175286.

4. —_ Pseudo-Laplaciens. II, Ann. Inst. Fourier (Grenoble) 33 (1983), 87-113.

5. J. Deshouillers, H. Iwaniec, R. Phillips, and P. Sarnak, Maass cusp forms, Proc. Nat. Acad. Sci. U.S.A. 82 (1985), 3533-3534.

6. D. Gilbarg and N. Trudinger, Elliptic partial differential equations of second order, Grundlehren der Math., bd. 224, Springer-Verlag, New York, 1977.

7. D. Hejhal, Regular b-groups, Degenerating Riemann surfaces and spectral theory, Mem. Amer. Math. Soc., vol. 88, Amer. Math. Soc., Providence, RI, 1990.

8. L. Ji, Spectral degeneration of hyperbolic Riemann surfaces, J. Differential Geom. 38 (1993), 263-314.

9. P. Lax and R. Phillips, Scattering theory for automorphic functions, Ann. of Math. Stud., no. 87, Princeton Univ. Press, Princeton, NJ, 1976.

10. R. Phillips and P. Sarnak, On cusp forms for cofinite subgroups of $\operatorname{PSL}(2, R)$, Invent. Math. 80 (1985), 339-364.

11. P. Sarnak, On cusp forms, Selberg Trace Formula and Related Topics, Contemp. Math., vol. 53, Amer. Math. Soc., Providence, RI, 1986, pp. 393-407.

12. A. Selberg, Harmonic analysis (Göttingen Lecture notes), Atle Selberg's Collected Papers, Springer-Verlag, Berlin and New York, 1989, pp. 626-674.

13. M. Wolf, Infinite energy harmonic maps and degeneration of hyperbolic surfaces, J. Differential Geom. 29 (1991), 487-539.

14. S. Wolpert, Asymptotics of the spectrum and the Selberg zeta function on the space of Riemann surfaces, Comm. Math. Phys. 112 (1987), 283-315.

15. _ The spectrum of a Riemann surface with a cusp, Taniguchi Symposium Lecture, November 1989, preprint.

16. __ Spectral limits for hyperbolic surfaces. I, Invent. Math. 108 (1992), 67-89.

17. __, Spectral limits for hyperbolic surfaces. II, Invent. Math. 108 (1992), 91-129.

18. __ Disappearance of cusp forms in special families, Ann. of Math. (2) (to appear).

Department of Mathematics, Massachusetts Institute of Technology, Cambridge, MASSACHUSETTS 02139

E-mail address: ji@math.mit.edu 